\title{
Intrinsic defects in nonstoichiometric $\beta$-SiC nanoparticles studied by pulsed magnetic resonance methods
}

\author{
D.V. Savchenko ${ }^{1,2}$, A. Pöppl ${ }^{2}$, E.N. Kalabukhova ${ }^{1}$, E.F. Venger ${ }^{1}$, M.P. Gadzira ${ }^{3}$, G.G. Gnesin ${ }^{3}$ \\ ${ }^{1}$ V. Lashkaryov Institute of Semiconductor Physics, NAS of Ukraine, \\ 45, prospect Nauky, 03028 Kyiv, Ukraine \\ Phone: +3(0-44)-525-62-97, fax: +3(0-44)-489-17-04; e-mail: katia@i.kiev.ua \\ ${ }^{2}$ Fakultät für Physik und Geowissenschaften, Universität Leipzig, \\ Linnéstr. 5, D-04103 Leipzig, Germany; tel: +49-341-973-26-08, fax: +49-341-973-26-49, \\ E-mail:poeppl7@physik.uni-leipzig.de \\ ${ }^{3}$ Institute for Problems of Materials Science, NAS of Ukraine, \\ 3, Krzhyzhanivskogo str., 03142 Kyiv, Ukraine, \\ Phone: +3(0-44)-424-24-71, fax: +3(0-44)-424-21-31, e-mail: dep14@ipms.kiev.ua
}

\begin{abstract}
Nonstoichiometric $\beta$-SiC nanoparticles (np-SiC) have been studied by electron paramagnetic resonance (EPR) and pulsed magnetic resonance methods including field swept electron spin echo (FS ESE), pulsed electron nuclear double resonance (ENDOR) and hyperfine sublevel correlation spectroscopy (HYSCORE). Four ESE signals related to the paramagnetic centers labeled D1, D2, D3, D4 with $g=2.0043, g=2.0029$, $g=2.0031, g=2.0037$ were resolved in FS ESE spectrum due to their different spin relaxation times. As deduced from the study of the superhyperfine structure of the D2 defect by FS ESE, pulse ENDOR and HYSCORE methods the dominant paramagnetic center is a carbon vacancy $\left(\mathrm{V}_{\mathrm{C}}\right)$ localized in $\beta$-SiC crystalline phase of the np-SiC. The parameters of the $\mathrm{D} 2$ center coincide with those found for the $\mathrm{V}_{\mathrm{C}}$ in np-SiC obtained by laser pyrolysis method. Three other defects were identified by comparison of their EPR parameters with the microstructure of the np-SiC. The D1 defect was attributed to the $\mathrm{V}_{\mathrm{C}}$ vacancy located in $\alpha$-SiC crystalline phase. The D3 defect is identified with the carbon dangling bonds located in the carbon excess phase. The D4 defect was assigned to a threefold-coordinated $\mathrm{Si}$ atom bonded with one nitrogen atom, resulting in the formation of the local bonding $\mathrm{Si}_{-} \mathrm{Si}_{2} \mathrm{~N}$ configuration in $\alpha-\mathrm{Si}_{3} \mathrm{~N}_{4}$ phase.
\end{abstract}

Keywords: SiC nanoparticles, EPR, ESE, ENDOR, HYSCORE.

Manuscript received 09.10.09; accepted for publication 22.10.09; published online 04.12.09.

\section{Introduction}

Silicon carbide nanoparticles (np-SiC) are important engineering material because of its good mechanical properties, good oxidation resistance, low coefficient of thermal expansion, high hardness, and refractoriness [1]. On the other hand, it was reported that $\mathrm{np}-\mathrm{SiC}$ and their composites revealed size-dependent phenomena such as a high electrical conductivity in annealed samples [2], broad photoluminescence response [3] as well as specific features of the charge carriers [4] and nonlinear optical properties [5].

The np-SiC previously investigated by multifrequency electron paramagnetic resonance (EPR) spectroscopy were synthesized by $\mathrm{CO}_{2}$ laser pyrolysis of gas mixture composed by silane $\left(\mathrm{SiH}_{4}\right)$ and acetylene
$\left(\mathrm{C}_{2} \mathrm{H}_{2}\right)$ [6]. Nuclear magnetic resonance measurements (NMR) have shown the coexistence of the crystalline polytypes 3C SiC (cubic), 6 $\mathrm{H} \mathrm{SiC} \mathrm{(hexagonal)} \mathrm{and} \mathrm{a}$ fraction of amorphous structure in $\mathrm{np}-\mathrm{SiC}$ obtained by laser pyrolysis method [7]. Microscopic measurements showed that the sizes of the nanocrystallites varied within the range 10 to $35 \mathrm{~nm}$ and sizes of the grains were distributed within 35-90 nm.

Multifrequency EPR spectroscopy revealed the existence of at least three different paramagnetic intrinsic defects [2]. Two of them have been assigned to carbon vacancies located in the crystalline phases of $\mathrm{SiC}$ particles, and the third one was assigned to dangling bonds in excess carbon phases [8].

Previous field swept electron spin echo (FS ESE) investigations of the nitrogen doped np-SiC allowed to 
resolve two ESE signals with electronic $g$ values $g=$ $2.0048 \pm 0.0006$ (DI) and $g=2.0036 \pm 0.0006$ (DII) that were attributed to the carbon vacancies located in the crystalline $\alpha$-phase (6H SiC) and $\beta$-phase (3C SiC) of the $\mathrm{SiC}$ particles, respectively [9].

The present work focuses on the FS ESE investigation of the intrinsic defects in $\mathrm{np}-\mathrm{SiC}$, obtained by self-propagating high-temperature synthesis (SHS) of carbon and highly active silicon with the aim to compare the types of the defects formed in $\mathrm{np}-\mathrm{SiC}$ prepared by different technological methods. To get more detail information about local environment of the defects, the pulsed electron nuclear double resonance (ENDOR) $[10,11]$ and hyperfine (hf) sublevel correlation spectroscopy (HYSCORE) [12] were applied, which allowed to measure weak super hyperfine (shf) couplings between the paramagnetic center and surrounding ligand nuclei.

The ESE spectroscopy that demonstrates a remarkable rise in the last decade is based on exciting the spin system with high-power pulses of microwave (mw) energy and then measuring the emission signal that is referred to as a spin-echo signal - the response of the spin system on pulse excitation. In an FS ESE experiment, a two-pulse Hahn echo sequence [13] is employed (Fig. 1a), and the resulting echo intensity is monitored as a function of the magnetic field. FS ESE has properties that can make it desirable over conventional continuous wave (cw) EPR in certain cases. In particular, the FS ESE method is capable to disentangle overlapping EPR lines by taking advantage of differences in the phase memory spin-relaxation time of the paramagnetic centers. In addition, the FS ESE method does not suffer from the magnetic field modulation effect, and as such, very broad EPR spectra can be easily detected.

Another important application of the ESE techniques is the measurement of nuclear spin transition or ENDOR frequencies. Here, the transient nature of the ESE technique has a great advantage, because it makes it possible to observe the ENDOR transitions over a much wider temperature range and with higher sensitivity than in $\mathrm{cw}$ ENDOR experiments. The reason is that the specific relationships among mw power, radiofrequency (rf) power and the various spin relaxation times, that are necessary for $\mathrm{cw}$ ENDOR, are not required in the pulsed operation mode.

The two most common pulse ENDOR methods were introduced by Mims [11] and Davies [10]. A good overview of pulsed ENDOR methodology can be found in [14]. In Mims ENDOR, $\pi / 2-\tau-\pi / 2$ is used to create polarization for the electron spin and a third $\pi / 2$ pulse

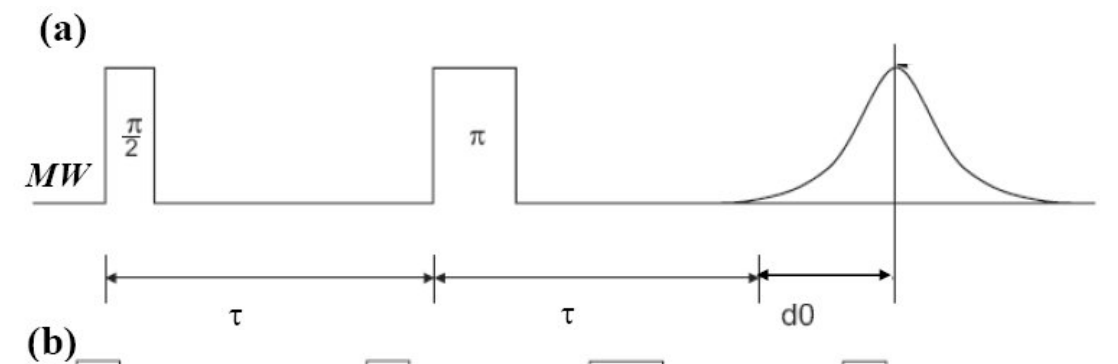

(b)
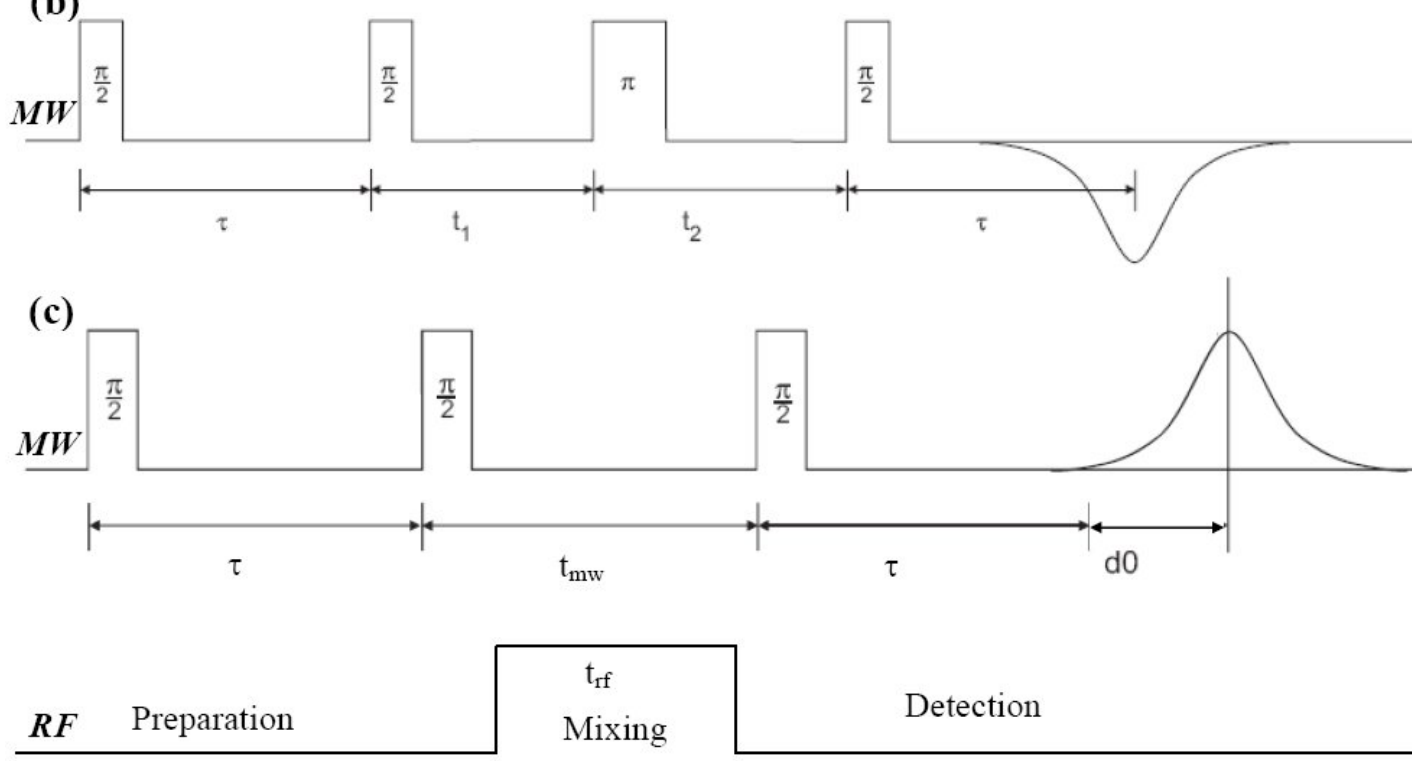

Fig. 1. Pulse sequences used in various experiments: (a) - two-pulse FS ESE sequence (d0 - detection trigger offset time), (b) - four-pulse (two dimensional) ESEEM (HYSCORE) sequence, (c) - Mims pulse ENDOR sequence. 
creates a stimulated echo at time $\tau$ (Fig. 1c). During the mixing period between the second and third mw pulses, a $\pi$ rf pulse is used to excite a nuclear spin transition, which leads to defocusing the electron spins and consequently a decrease in the observed stimulated electron spin echo. The frequency of this rf pulse is varied and the stimulated echo intensity is monitored. The resulting spectrum is an NMR spectrum of the nuclear spins in the vicinity of the electron being probed.

HYSCORE (Fig. 1b) is a two-dimensional fourpulse electron-spin-echo envelope-modulation (ESEEM) technique where a series of microwave ( $\mathrm{mw}$ ) pulses is applied to the spin system and the times $t_{1}$ and $t_{2}$ between the pulses are scanned. In addition to the decay of the four-pulse electron spin echo, which results from relaxation processes, modulation may be present from nuclei in the close environment about the paramagnetic center having a nuclear spin larger than zero. The frequencies of the modulations are identical to those observed in the ENDOR spectra, but the ESEEM and HYSCORE techniques have the advantage over ENDOR that additional information concerning the hf interaction with surrounding magnetic nuclei can be retrieved from the echo modulation amplitudes. In HYSCORE, after Fourier transformation, off-diagonal peaks are observed which correlate two frequencies of the same nucleus, similar to two-dimensional NMR spectroscopy. A crosspeak correlates a nuclear transition frequency of one electron spin sublevel $\mathrm{M}_{s}$ to a nuclear transition frequency of the same nucleus but of the other $\mathrm{M}_{s}$ sublevel. It is important to note that correlations between transitions of different nuclei coupled to the same paramagnetic center are much lower in intensity. In principle, in the case of anisotropic EPR spectra, information about the full hf tensors of the various magnetic nuclei can be elucidated from orientationselective HYSCORE spectra in combination with spectral simulations even for disordered samples. A comprehensive description of pulsed EPR spectroscopy is given in the book of Schweiger and Jeschke [14].

\section{Experiment}

\subsection{Sample preparation and characterization}

The np-SiC was prepared by self-propagating high temperature synthesis (SHS) method in argon atmosphere from a mixture of fine elemental silicon powder and thermally exfoliated graphite (TEG) [1517]. The hetero-phase interaction between components is accompanied by an exothermic effect. The ignition temperature was in the range of $1200-1250{ }^{\circ} \mathrm{C}$. The reaction product is $\beta$-SiC in agglomerated state which could be easily reduced to nanoparticles. The unreacted carbon in the product after synthesis was removed by heating in air at $700{ }^{\circ} \mathrm{C}$. The final clean-up to remove $\mathrm{SiO}_{2}$ was carried out by etching in $\mathrm{HF}$, rinsing in distilled water and drying. The investigation of the microstructure of the $\mathrm{np}$-SiC by X-ray diffractometry (XRD) and high resolution transmission electron microscopy (HRTEM) methods have shown that the synthesis of the np-SiC in non-equilibrium conditions leads to the decrease of the $\beta$-SiC lattice parameter up to $4.3536 \AA$ in comparison with the standard value $(4.3589 \AA)$. A lower value of the lattice parameter in comparison with a standard one can be explained by the partial substitution of silicon atoms in SiC lattice by carbon, nitrogen and oxygen atoms having smaller covalent radii [15] resulting in the formation of the solid solution $\mathrm{SiC}-\mathrm{C}$ and small fraction of the $\mathrm{Si}_{3} \mathrm{~N}_{4}, \mathrm{Si}_{2} \mathrm{~N}_{2} \mathrm{O}$, $\mathrm{SiO}_{2}$ phases. Fig. 2 shows HRTEM image of the np-SiC with the average size of the particles of about $50 \mathrm{~nm}$.

Fig. 3 shows Raman spectrum (RS) of the np-SiC powder and standard crystalline $\beta$-SiC sample which were obtained using $514.5 \mathrm{~nm}$ Ar-laser radiation excitation. RS were taken with a double grating spectrometer at room temperature. The signal was registered with a cooled phototube operating in the photon counting mode in back scattering geometry. Analysis of RS peak frequencies observed in $\mathrm{np}-\mathrm{SiC}$ showed that np-SiC should be attributed to the $3 \mathrm{C}$ polytype. As was seen from Fig. 3, the RS of the np-SiC is characterized by the sharp peak at $790 \mathrm{~cm}^{-1}$ that corresponds to the transversal (TO) phonon band of $\beta$ $\mathrm{SiC}[18]$.

\subsection{Spectroscopic measurements}

X-band FS ESE, pulsed ENDOR and HYSCORE experiments have been performed on Bruker ESP 380 and ELEXYS E580 spectrometers at $6 \mathrm{~K}$. The Q-band cw EPR spectra were measured on RE1308 spectrometer at $T=4 \mathrm{~K}$. The FS ESE spectra simulations were performed using Easyspin toolbox [19]. An overview of the specific pulse sequences used in the various experiments is given in Fig. 1.

The X-band FS ESE spectra were measured with a two-pulse echo sequence (Fig. 1a) with mw pulse lengths: $t_{\pi / 2}=16 \mathrm{~ns}$ and $t_{\pi}=32 \mathrm{~ns}$ with the pulse delay $\tau=400 \mathrm{~ns}$ (short pulses) and $t_{\pi / 2}=96 \mathrm{~ns}$ and $t_{\pi}=192 \mathrm{~ns}$ with the pulse delay $\tau=800 \mathrm{~ns}$ (long pulses).

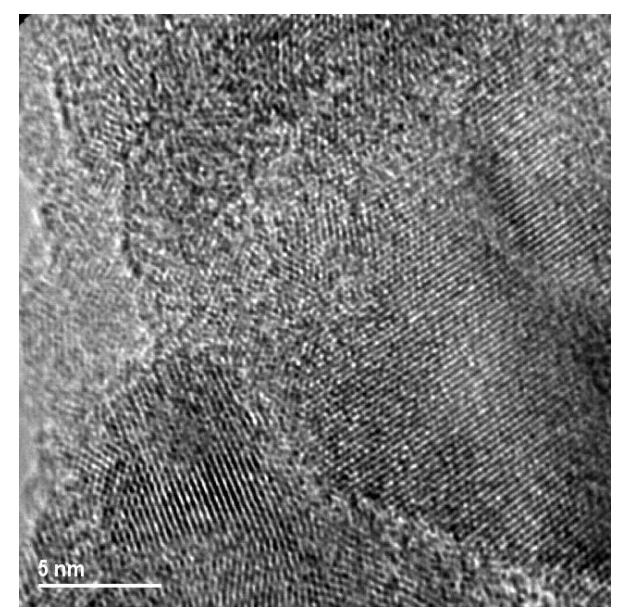

Fig. 2. HRTEM image of the np-SiC particles synthesized under non-equilibrium conditions. 


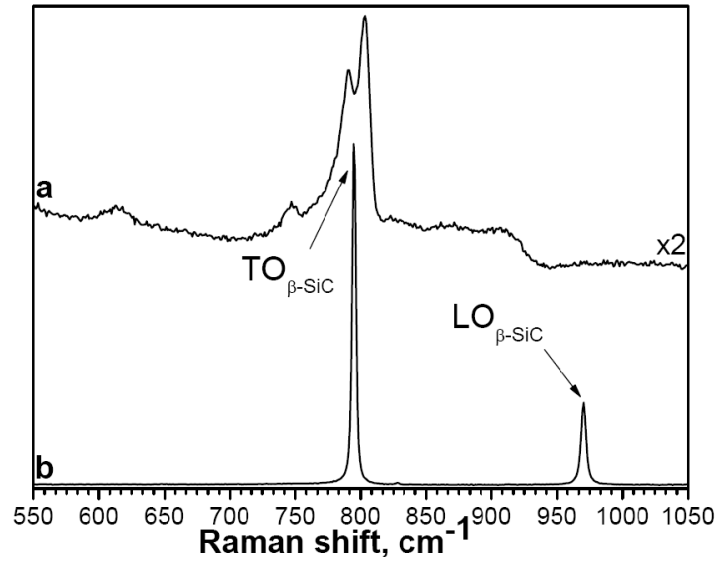

Fig. 3. Raman spectra of $n p-S i C$ (a) and standard $\beta-\mathrm{SiC}(b)$. The np-SiC sample was annealed in air at $700{ }^{\circ} \mathrm{C}$, and was rinsed from $\mathrm{SiO}_{2}$ by etching in $\mathrm{HF}$.

For the X-band Mims ENDOR experiments the stimulated electron spin echo sequence $\pi / 2-\tau-\pi / 2-t_{m w^{-}}$ $\pi / 2-\tau$-echo was used (see Fig. 1c), with nonselective mw $\pi / 2$ pulse lengths of $t_{\pi / 2}=48 \mathrm{~ns}$ and the pulse delay $\tau=560$ ns. A selective radio frequency (rf) pulse of variable frequency and length of $t_{r f}=12 \mu \mathrm{s}$ was applied between the second and third mw pulses.

HYSCORE experiments [13] were recorded using nonselective mw pulses of $t_{\pi / 2}=16 \mathrm{~ns}$ and $t_{\pi}=32 \mathrm{~ns}$ with the pulse delays of $\tau=136 \mathrm{~ns}$ and $104 \mathrm{~ns}$ between first two $\pi / 2 \mathrm{mw}$ pulses (see Fig. 3b). In total, $170 \times 170$ data points were acquired for $t_{1}$ and $t_{2}$, and the spectra with the different $\tau$ values were added prior twodimensional (2D) Fourier transformation (FT) and finally 2D FT magnitude spectra were presented.

\section{Results}

The cw EPR spectrum of the np-SiC measured at Q-band frequency and $4.2 \mathrm{~K}$ was depicted in Fig. 4 . It is seen that EPR spectrum consists of several unresolved EPR signals with central $g$-factor $\sim 2.0025$. Taking into account the advantage of the pulse EPR technique over the cw EPR (the absence of the magnetic field modulation effect on the EPR linewidth), we applied pulse EPR methods to resolve these EPR signals. Fig. 5 shows the first derivative of the X-band FS ESE spectrum in np-SiC that consists of a few signal components. As was seen from Fig. 5, the FS ESE spectra taken with different pulse delays $\tau$ have different intensity ratios between signal components indicating that the components have different electron spin phase memory relaxation times and can be assigned to a few distinct intrinsic defects. The parameters of the four paramagnetic centers given in Table 1 and denoted as D1, D2, D3 and D4 were obtained by deconvolution of the experimental FS ESE spectrum. It was found that the $g$ values of the D1, D2 and D3 centers are close to those for DI, DII and DIII centers observed in np-SiC obtained by laser pyrolisis method [2].
Table 1. Parameters of paramagnetic centers observed in two-pulse FS ESE spectra at $T=6 \mathrm{~K}\left(\Delta H_{p p}\right.$ is the peak-topeak EPR linewidth).

\begin{tabular}{|c|c|c|c|c|}
\hline Center & $g_{x x}$ & $g_{y y}$ & $g_{z z}$ & $\begin{array}{c}\Delta H_{p p}, \\
\mathrm{mT}\end{array}$ \\
\hline D1 & $2.0041(2)$ & $2.0041(2)$ & $2.0041(2)$ & 0.24 \\
\hline D2I & $2.0029(2)$ & $2.0029(2)$ & $2.0029(2)$ & 0.24 \\
\hline D3 & $2.0031(2)$ & $2.0031(2)$ & $2.0031(2)$ & 1.8 \\
\hline D4 & $2.0036(2)$ & $2.0036(2)$ & $2.0036(2)$ & 0.6 \\
\hline
\end{tabular}

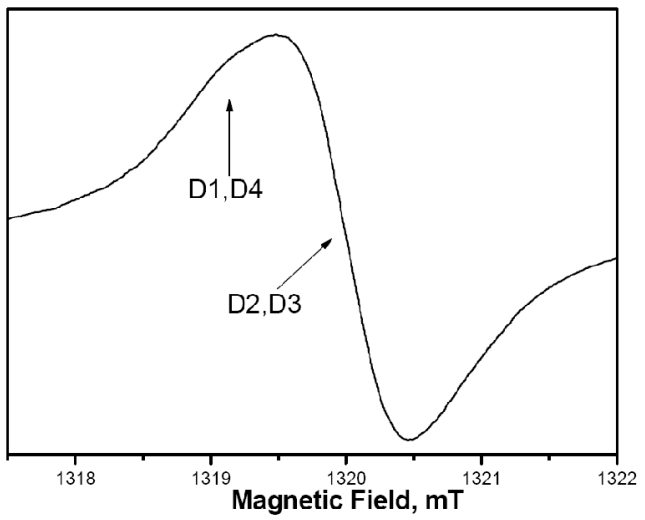

Fig. 4. The Q-band cw EPR spectrum measured on the np-SiC sample at $T=4 \mathrm{~K}$.

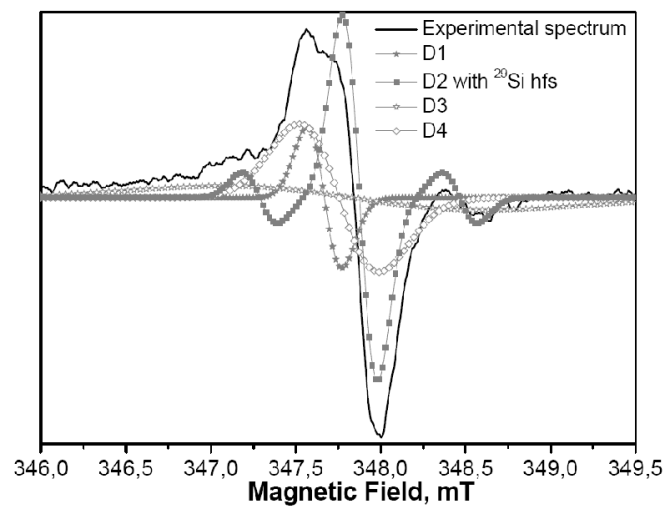

(a)

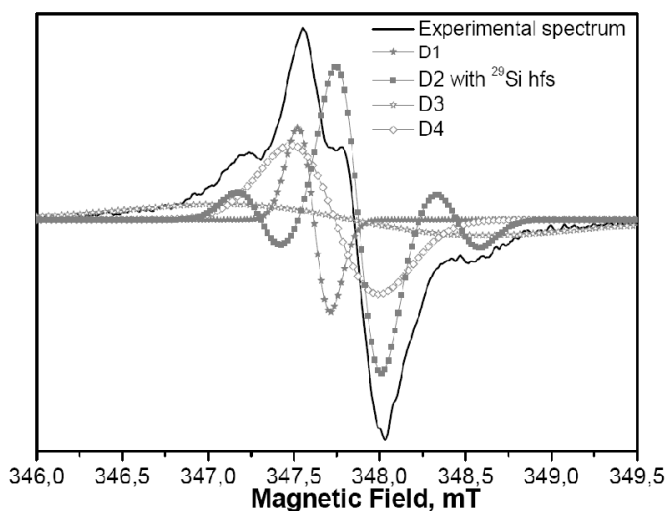

(b)

Fig. 5. First derivative of $X$-band FS ESE spectrum measured using the np-SiC sample at $T=6 \mathrm{~K}$ with short pulses (a) and long pulses (b). 
Additional shoulders observed in the wings of the first derivative of the FS ESE spectrum (Fig. 5) indicate the presence of a isotropic shf splitting $\left(A_{\text {iso }}=29.7 \mathrm{MHz}\right)$ with surrounding ${ }^{29} \mathrm{Si}$ or ${ }^{13} \mathrm{C}$ nuclei, both isotopes having a nuclear spin $I=1 / 2$ and natural abundances of 4.7 and $1.1 \%$. The shf lines are symmetric with respect to the signal D2 with $g=2.0029$ and can be consequently assigned to the D2 center. The intensity ratio between central line and satellites is about 9-10\%, which corresponds to the natural abundance of two equivalent ${ }^{29} \mathrm{Si}$ atoms. The defect with the same shf splitting and $g=2.0036 \pm 0.0006$ was previously observed in np-SiC obtained by the laser pyrolisis method and was assigned with to the carbon vacancy [9]. Therefore, it can be concluded that D2 center is also related to the carbon vacancy.

The identification of the other intrinsic defects was undertaken by comparison of their experimental EPR parameters with the microstructure of the $\mathrm{np}-\mathrm{SiC}$ materials. The feature of the D1 centers was found to be consistent with that of DI centers with $g=2.0048$ \pm 0.0006 observed in $\mathrm{np}-\mathrm{SiC}$ obtained by the laser pyrolisis method. It was shown that DI centers disappears progressively with decreasing the degree of hexagonality of the np-SiC, which is favoured by annealing the sample [8]. Therefore, the observed small fraction of the D1 center compared to D2 center in np$\mathrm{SiC}$ which have mostly cubic crystalline structure indicates that D1 center is the intrinsic defect localized in the hexagonal phase of np-SiC.

The $g$-factor and linewidth of the D3 center coincide with those of DIII center observed in $\mathrm{np}-\mathrm{SiC}$ obtained by the laser pyrolisis method $[2,8]$. It was proposed that the DIII is related with carbon dangling bonds located in the carbon excess phase of np-SiC $[2,8]$. Taking into account that the SiC-C solid solution coexists with $\beta$-SiC phase in crystallite structure of np$\mathrm{SiC}$, we may conclude that the D3 center also originates from $s p^{3}$-coordinated carbon dangling bonds located in the carbon excess phase of $\mathrm{np}-\mathrm{SiC}$.

The FS ESE spectrum of the D4 center is characterized by the isotropic $g$ factor $g=2.0037$ \pm 0.0002 , linewidth $(6 \pm 0.5) \mathrm{Gs}$, and Gaussian line shape. The parameters of the D4 defect are typical for the bulk $K$-center previously observed in amorphous hydrogenated $\mathrm{Si}_{3} \mathrm{~N}_{4}$ layers [20]. The study of this defect in silicon nitride layers of different compositions $\mathrm{Si}_{\mathrm{x}} \mathrm{N}_{\mathrm{y}}$ has shown that the $g$-factor of the $K$-center is dependent on the local bonding configuration $\mathrm{Si}-\mathrm{X}_{3}$ and varies from 2.0030 for $\mathrm{Si}-\mathrm{N}_{3}$ to 2.0040 for $\mathrm{Si}_{-} \mathrm{NSi}_{2}$ [20]. The linewidth depends also on the configuration due to the unresolved shf interaction with the ${ }^{14} \mathrm{~N}(\mathrm{I}=1)$ nuclei. Thus, considering that X-ray investigations confirms the existence of the peak reflections due to $\alpha-\mathrm{Si}_{3} \mathrm{~N}_{4}$ and $\alpha-\mathrm{Si}_{2} \mathrm{~N}_{2} \mathrm{O}$ phases in $\mathrm{np}-\mathrm{SiC}$, the D4 center can be assigned to the defect located in $\alpha$ $\mathrm{Si}_{3} \mathrm{~N}_{4}$ phase with $\mathrm{Si}-\mathrm{NSi}_{2}$ configuration.

The detailed study of the ligand structure of the intrinsic defects by the pulse ENDOR and HYSCORE methods was concentrated on the D2 center. Other defects have an insufficient intensity of the FS ESE signals to be investigated by pulse ENDOR and HYSCORE methods.

Pulsed ENDOR and HYSCORE spectra were recorded at the FS ESE position of D2 center. Fig. 6 shows ENDOR spectrum measured at $T=6 \mathrm{~K}$ using the Mims pulse sequence, which is more sensitive for measuring small hf couplings, if the phase memory time $T_{M}$ of the sample is sufficiently long to allow an optimal $\tau$ value to be used. For large hf couplings, it is usually preferable to employ the Davies ENDOR sequence with a well chosen length for the inversion mw pulse [14].

The ENDOR spectra proved to be isotropic and are dominated by a few doublets centered at the ${ }^{1} \mathrm{H}$ Larmor frequency. Therefore, to understand the ENDOR results we will consider the isotropic shf interaction (Fermi contact term). The interaction constant $a_{i}$ reflects the spin density of the donor electron wave function $(\Psi)$ at the site of the nucleus $\left(r_{i}\right)$ and is given by:

$a_{i}=\frac{8 \pi}{3} \mu_{B} \mu_{n} g_{e} g_{n i}\left|\Psi\left(r_{i}\right)\right|^{2}$

where $\mu_{B}$ is the electron Bohr magneton, $\mu_{n}$ - nuclear magneton, $g_{e}-g$-factor of free electron, $g_{n i}$ is the $g_{n}$ factor of the nucleus $i, g_{n}$ - nuclear $g$-factor.

The related ENDOR transitions for a nuclear spin $\mathrm{I}=1 / 2 \quad$ (e.g. $\left.{ }^{1} \mathrm{H},{ }^{13} \mathrm{C},{ }^{29} \mathrm{Si}\right)$ have the following frequencies [21]:

$v_{\text {ENDOR } i}=\frac{1}{h}\left|g_{n i} \mu_{n} B \pm \frac{a_{i}}{2}\right|$,

where $B$ is the external magnetic field.

Therefore, the expression (2) predicts that each nucleus $i$ will give rise to two ENDOR transitions symmetrically placed around their Zeeman frequency $g_{n i} \mu_{n} B_{0} / h$ (if the weak coupling limit

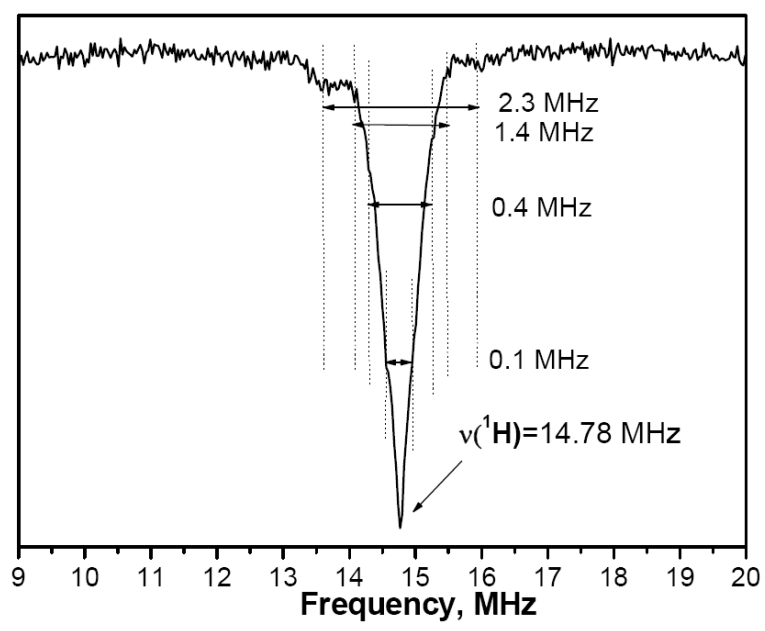

Fig. 6. X-band pulsed ENDOR measured on D2 FS ESE position in the np-SiC sample at $T=6 \mathrm{~K}$ using Mims pulse ENDOR sequence. 
frequency has further been analyzed by HYSCORE spectroscopy that has a much better sensitivity that ENDOR in this frequency range.

As was seen from Fig. 7b, the low frequency part of the HYSCORE spectrum is dominated by an intense ${ }^{29} \mathrm{Si}$ cross peak ridge $([0.64 ; 5.33],[5.33 ; 0.64])$ with a total width $4.69 \mathrm{MHz}$. Therefore, according to [9] the paramagnetic center D2 couples to the ${ }^{29} \mathrm{Si}$ nucleus located in the next neighborhood of the D2 center with a maximum shf interaction $A_{\max }=4.69 \mathrm{MHz}$ corresponding to silicon nuclei denoted as $\mathrm{Si}(\mathrm{IV})$ in [9]. The detailed analysis of widths of the ridges and the maximum shift of the cross peak ridges given in [9] allows to evaluate the range of the isotropic $\left(A_{i s o}^{\mathrm{Si}(\mathrm{IV})}\right)$ and anisotropic $\left(T^{\mathrm{Si}(\mathrm{IV})}\right) \operatorname{shf}$ coupling limits for $\mathrm{Si}(\mathrm{IV})$ : $1.6 \mathrm{MHz} \leq A_{i s o}^{\mathrm{Si}(\mathrm{IV})} \leq 3.4 \mathrm{MHz}$ and $1.4 \mathrm{MHz} \geq T^{\mathrm{Si}(\mathrm{IV})} \geq 0.5 \mathrm{MHz}$.

The ridge at ${ }^{13} \mathrm{C}$ Larmor frequency ([2.85; 4.44], [4.44; 2.85]) in HYSCORE spectrum is less intensive than that observed for ${ }^{29} \mathrm{Si}$, and its width corresponds to a maximum carbon shf interaction $A_{\max }=1.59 \mathrm{MHz}$ denoted as carbon nuclei C(II) in [9]. Thus, we can conclude that we observe approximately the same value for ${ }^{29} \mathrm{Si}$ and ${ }^{13} \mathrm{C}$ hf structure, as it was observed in [9] for DII center. In our case analogous to [9], the maximum shift of the cross peak ridges is below the spectral resolution $0.06 \mathrm{MHz}$ of the experiment.

Except for the intense peaks due to ${ }^{29} \mathrm{Si}$ and ${ }^{13} \mathrm{C}$, a weak ${ }^{1} \mathrm{H}$ cross peak ridge $([14.23 ; 15.46],[15.46$; 14.23]) with a maximum spread of about $1.22 \mathrm{MHz}$ is observed at the proton nuclear Larmor frequency (Fig. 7a). The obtained value is close to the proton shf coupling value $A_{2}^{\mathrm{H}}=1.4 \mathrm{MHz}$ as already deduced from the Mims ENDOR spectrum. To explain the absence of the ${ }^{1} \mathrm{H}$ cross peak ridges corresponding to the protons with $A_{1}^{\mathrm{H}}=2.3 \mathrm{MHz}$ in $2 \mathrm{D}$ spectrum, we can propose that ENDOR lines are broadened due to distribution in the isotropic shf interaction, while the anisotropic coupling is negligible.

\section{Discussion}

With the aim to compare the type of the intrinsic defects formed in $\mathrm{np}-\mathrm{SiC}$ obtained by different technology methods, the investigation of the nonstoichiometric np$\mathrm{SiC}$ prepared by SHS of carbon and highly active silicon was performed by EPR, FS ESE, ENDOR and HYSCORE methods. FS ESE measurements of the np$\mathrm{SiC}$ prepared by SHS method revealed four types of the paramagnetic centers. Three of them (D1, D2, D3) have quite similar parameters to those observed for the intrinsic defects in $\mathrm{np}-\mathrm{SiC}$ obtained by laser pyrolisis method. The ligand structure of the D2 center observed in FS ESE, ENDOR and HYSCORE spectra is found to be consistent with that observed for DII center in $n p-$ $\mathrm{SiC}$, obtained by laser pyrolisis method. Good agreement between the value of the largest shf splitting

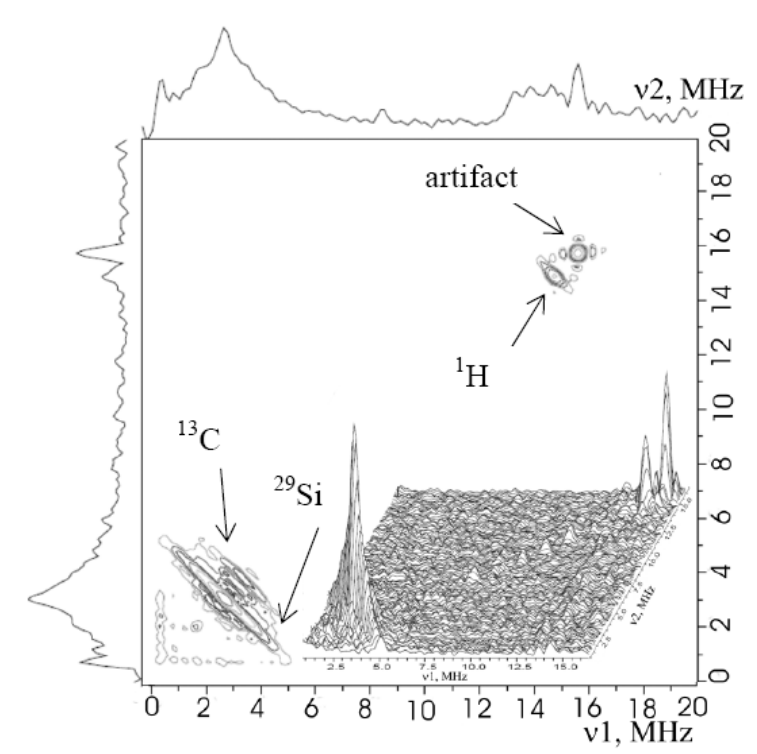

(a)

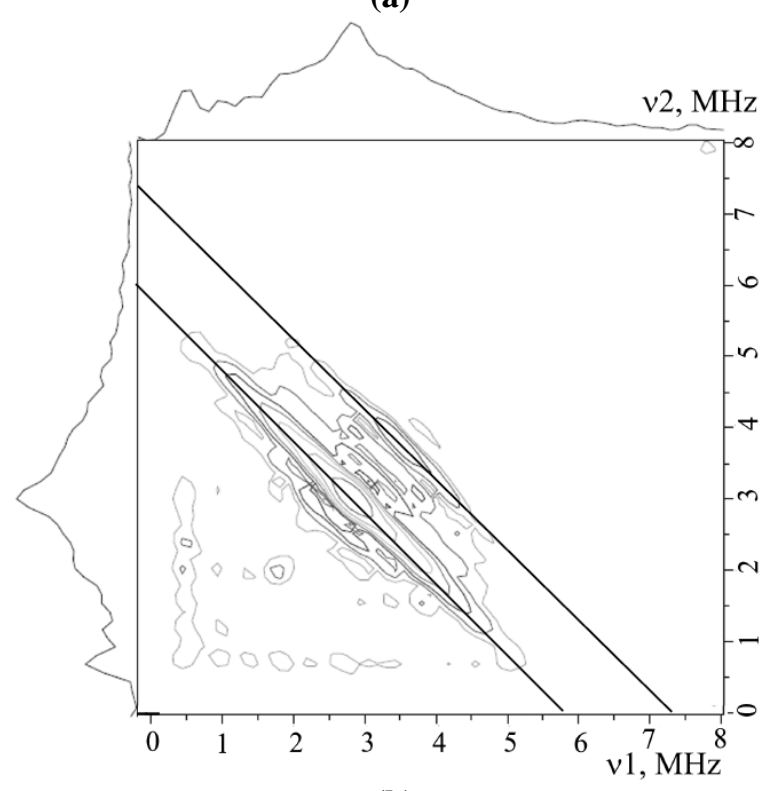

(b)

Fig. 7. Contour plot of the HYSCORE spectrum for np-SiC measured on D2 FS ESE position. (a) the full spectral range, insertion is the stacked plot of the HYSCORE spectrum; (b) low frequency part. The solid lines in (b) indicate the $v_{1}=-v_{2}$ axes at the ${ }^{29} \mathrm{Si}$ and ${ }^{13} \mathrm{C}$ Larmor frequency.

of the D2 defect observed in FS ESE spectrum and theoretically evaluated shf constant for carbon vacancy in $\mathrm{np}-\mathrm{SiC}$ performed in [22] by self-consistent normconserving pseudopotential band energy calculations indicates that the D2 center and likewise the DII center can be assigned to a carbon vacancy. It is necessary to add that the corresponding shf constants of ${ }^{29} \mathrm{Si}$ for carbon vacancies in bulk $\mathrm{SiC}$ crystals are essentially higher [23] indicating that the wavefunction of the unpaired electron of the vacancy in $\mathrm{np}-\mathrm{SiC}$ is substantially higher delocalized than in bulk material. At 
the same time, considering that the ENDOR spectrum of the D2 center shows a weak shf interaction with hydrogen we may suggest that the carbon vacancy environment would be contaminated with hydrogen forming the hydrogen-related defect $\left(\mathrm{V}_{\mathrm{C}}+\mathrm{H}\right)$ with donorlike behavior $[24,25]$. Table 2 shows the calculated shf interaction for carbon vacancies in $\mathrm{np}-\mathrm{SiC}$ and hydrogenated carbon vacancy with $\mathrm{S}=1 / 2$ in bulk $\mathrm{SiC}$ calculated in the framework of density functional theory (DFT) in [25] along with experimental shf interaction observed for D2 and DII center. As was seen from Table 2, the ratio between shf interaction with hydrogen and ${ }^{29} \mathrm{Si}$ observed for D2 and DII center are not in contradiction with that theoretically predicted for the hydrogenated carbon vacancy in SiC bulk crystals. Furthermore, the configuration with an $\mathrm{H}$-atom in the center of three ligands $\mathrm{Si}_{3}, \mathrm{Si}_{4}$ and $\mathrm{Si}_{2}$ provides nearly isotropic shf parameters for the case of motional averaged structure of the complex caused by a dynamic Jahn-Teller effect, which is in reasonable agreement with the negligible value of the anisotropic coupling of the hydrogen observed in the ENDOR spectrum of the D2 center at $T=4 \mathrm{~K}$. Strongly anisotropic ${ }^{1} \mathrm{H}$-related shf splitting was predicted only for $T=0 \mathrm{~K}$. To confirm the assumed contamination of the carbon vacancies with hydrogen, further theoretical calculations of the $\left(\mathrm{V}_{\mathrm{C}}+\mathrm{H}\right)$ complex in $\mathrm{np}-\mathrm{SiC}$ are required.

Other defects cannot be conclusively identified from the experimental data because of the lack of the ligand structure in their FS ESE spectrum. But comparison between microstructure of the $\mathrm{np}-\mathrm{SiC}$ and obtained experimental EPR parameters of the D1 center enables to attribute it to the intrinsic defect located in crystalline hexagonal phase of np-SiC. As was shown in [22], the vacancies are a necessary attribute of the crystalline phase of the np-SiC. Therefore, we may conclude that the D1 center like to the D2 and DI centers is caused by a charged vacancy.

Taking into account that the $\mathrm{SiC}-\mathrm{C}$ solid solution coexists with $\beta$-SiC phase in crystallite structure of the np$\mathrm{SiC}$, it was concluded that the D3 center like to the DIII center originates from $s p^{3}$-coordinated carbon dangling bonds located in the carbon excess phase of the np-SiC.

Table 2. The comparison of calculated shf interaction for carbon vacancies in np-SiC [22] and hydrogenated carbon vacancy in bulk $\mathrm{SiC}$ [25] $(\mathrm{S}=1 / 2)$ with experimental shf interaction observed for D2 and DII center.

\begin{tabular}{|c|c|c|c|c|c|}
\hline Ligands & \multicolumn{2}{|c|}{${ }^{29} \mathrm{Si}$} & \multicolumn{2}{|c|}{${ }^{1} \mathrm{H}$} & \multirow[t]{2}{*}{ Ref. } \\
\hline Defect & $\begin{array}{l}A_{\text {iso }}, \\
\mathrm{MHz}\end{array}$ & $\begin{array}{c}T, \\
\mathrm{MHz}\end{array}$ & $\begin{array}{l}A_{\text {iso }}, \\
\mathrm{MHz}\end{array}$ & $\begin{array}{c}T \\
\mathrm{MHz}\end{array}$ & \\
\hline $\begin{array}{l}\text { Calcul. } V_{C} \\
\text { for np-SiC }\end{array}$ & 32.88 & -4.35 & - & - & {$[22]$} \\
\hline $\begin{array}{c}\text { Calcul. } \\
\mathrm{V}_{\mathrm{C}}+\mathrm{H}\end{array}$ & $\begin{array}{c}-173.98 \\
\left(\mathrm{Si}_{2-4}\right)\end{array}$ & $\begin{array}{l}-19.7 \\
\left(\mathrm{Si}_{2-4}\right)\end{array}$ & 5.91 & 0.66 & \multirow{2}{*}{ [25] } \\
\hline $\begin{array}{l}\mathrm{H} \text { between } \\
\mathrm{Si}_{2}, \mathrm{Si}_{3}, \mathrm{Si}_{4}\end{array}$ & $-5.6 \mathrm{Si}_{1}$ & $-5.6 \mathrm{Si}_{1}$ & - & - & \\
\hline $\begin{array}{c}\text { Exp. D2 in } \\
\text { np-SiC }\end{array}$ & 29.7 & - & 2.3 & - & $\begin{array}{l}\text { This } \\
\text { work }\end{array}$ \\
\hline
\end{tabular}

\begin{tabular}{|c|c|c|c|c|c|}
\hline $\begin{array}{c}\text { Exp. DII in } \\
\text { np-SiC }\end{array}$ & 29.7 & - & 6.4 & - & [9] \\
\hline
\end{tabular}

The D4 center characterized by $g=2.0037$ \pm 0.0002 and a Gaussian line shape was attributed to the bulk intrinsic defect located in $\alpha-\mathrm{Si}_{3} \mathrm{~N}_{4}$ phase of the np$\mathrm{SiC}$ with $\mathrm{Si}-\mathrm{NSi}_{2}$ configuration.

Thus, the structural phases involved into the np-SiC are characterized by the intrinsic defects that are necessary for thermodynamic stabilization of the particle size and structure [22]. Crystalline cubic and hexagonal phases are monitored by the bulk defect like to a charged vacancy, while carbon excess phase of the $\mathrm{np}-\mathrm{SiC}$ is stabilized by presence of dangling bonds.

\section{Acknowledgments}

D.V. Savchenko and A. Pöppl acknowledge financial support by the DFG (grant PO 426/6-1).

Authors are grateful to the Joint Use Center of Scientific Equipment "EPR spectroscopy", NAS of Ukraine.

\section{References}

1. K. Niihara, A. Nakahira, Strengthening and toughening mechanisms in nanocomposite ceramics // Ann. Chim. Fr. 16(4), p. 479-486 (1991).

2. S. Charpentier, A. Kassiba, J. Emery, M. Cauchetier, Investigation of the paramagnetic centres and electronic properties of silicon carbide nanomaterials // J. Phys.: Condens. Matter 11(25), p. 4887-4897 (1999).

3. A. Kassiba, M. Makowska-Janusik, J. Boulé, J.F. Bardeau, A. Bulou, N. Herlin-Boime, Photoluminescence features on the Raman spectra of quasi-stoichiometric $\mathrm{SiC}$ nanoparticles: Experimental and numerical simulations // Phys. Rev. B 66(15), 155317-15323 (2002).

4. A. Kassiba, W. Bednarski, A. Pud et al., Hybrid core-shell nanocomposites based on silicon carbide nanoparticles functionalized by conducting polyaniline: electron paramagnetic resonance investigations // J. Phys. Chem. C 111(31), p. 11544-11551(2007).

5. I.V. Kityk, M. Makowska-Janusik, A. Kassiba, K.J. Plucinski, SiC nanocrystals embedded in oligoetheracrylate photopolymer matrices; new promising nonlinear optical materials // Opt. Mater. 13(4), p. 449-453 (2000).

6. M. Cauchetier, O. Croix, M. Luce, Laser synthesis of silicon carbide powders from silane and hydrocarbon mixtures // Adv. Ceram. Mater. 3(6), p. $548-552$ (1988).

7. M. Tabellout, A. Kassiba, S. Tkaczyk, L. Laskowski, J. Swiatek, Dielectric and EPR investigations of stoichiometry and interface effects in silicon carbide nanoparticles // J. Phys.: Condens. Matter 18(4), p. 1143-1155 (2006). 
8. A. Kassiba, M. Makowska-Janusik, J. Boucle, J.F. Bardeau, A. Bulou, N. Herlin, M. Mayne, $\mathrm{X}$. Armand, Stoichiometry and interface effects on the electronic and optical properties of $\mathrm{SiC}$ nanoparticles // Diamond and Relat. Mater. 11(36), p. 1243-1247 (2002).

9. D.V. Savchenko, A. Pöppl, J. Hoentsch, E.N. Kalabukhova, Y. Bulois and A. Kassiba, Intrinsic defects in $\mathrm{SiC}$ nanoparticles as studied by pulsed electron paramagnetic resonance // Solid State Communs. 146(1-2), p. 83-87 (2008).

10. E.R. Davies, A new pulse ENDOR technique // Phys. Lett. A 47(1), p. 1-2 (1974).

11. W.B. Mims, Pulsed ENDOR experiments // Proc. Roy. Soc. London A 283(1395), p. $452-457$ (1965).

12. P. Höfer, A. Grupp, H. Nebenführ and M. Mehring, Hyperfine sublevel correlation (HYSCORE) spectroscopy: A 2D ESR investigaton of the squaric acid radical // Chem. Phys. Lett. 132(3), p. 279-282 (1986).

13. E.L. Hahn, Spin Echoes // Phys. Rev. 80(4), p. 580594 (1950).

14. A. Schweiger, G. Jeschke, Principles of Pulse Electron Paramagnetic Resonance. Oxford University Press, Oxford, 2001.

15. M. Gadzira, G. Gnesin, O. Mykhaylyk, V. Britum, O. Andreyev, Solid solution of carbon in $\beta-\mathrm{SiC} / /$ Mater. Lett. 35(5-6), p. 277-282 (1998).

16. O.O. Mykhaylyk, M.P. Gadzira, Arrangement of C atoms in the SiC-C solid solution // Acta Cryst. B 55(3), p. 297-305 (1999).

17. M. Gadzira, G, Gnesin, O. Mykhaylyk, O. Andreyev, Synthesis and structural peculiarities of nonstoichiometric $\beta$-SiC // Diamond and Relat. Mater. 7(10), p. 1466-1470 (1998).
18. D.W. Feldman, J.H. Parker Jr., W.J. Choyke, L. Patrick, Phonon dispersion curves by Raman scattering in $\mathrm{SiC}$, polytypes $3 \mathrm{C}, 4 \mathrm{H}, 6 \mathrm{H}, 15 \mathrm{R}$, and 21R // Phys. Rev. 173(3), p. 787-793 (1968).

19. S. Stoll, A. Schweiger, EasySpin, a comprehensive software package for spectral simulation and analysis in EPR // J. Magn. Reson. 178(1), p. 42-55 (2006).

20. P. Aubert, H.J. von Bardeleben, F. Delmotte, J.L. Cantin, M.C. Hugon, Electron-paramagneticresonance study of the $(100) \mathrm{Si} / \mathrm{Si}_{3} \mathrm{~N}_{4}$ interface // Phys. Rev. B 59(16), p. 10677-10684 (1999).

21. J.-M. Spaeth, H. Overhof, Point Defects in Semiconductors. Springer-Verlag, Berlin Heidelberg, 2003.

22. I.V. Kityk, A. Kassiba K. Tuesu, C. Charpentier, Y. Ling, M. Makowska-Janusik, Vacancies in SiC nanopowders // Mater. Sci. Eng. B 77(2), p. 147158 (2000).

23. M. Bockstedte, M. Heid, O. Pankratov, Signature of intrinsic defects in SiC: $A b$ initio calculations of hyperfine tensors // Phys. Rev. B 67(19), 193102-1193102-4 (2003).

24. A. Gali, P. Deák, N.T. Son, E. Janzén, H.J. von Bardeleben, J.L. Monge, Calculation of hyperfine constants of defects in $4 \mathrm{H}-\mathrm{SiC} / /$ Mat. Sci. Forum 433-436, p. 511-514 (2003).

25. E.N. Kalabukhova, S.N. Lukin, D.V. Savchenko, W.C. Mitchel, S. Greulich-Weber, E. Rauls, U. Gerstmann, Possible role of hydrogen within the so-called X-center in semi-insulating 4H-SiC // Mat. Sci. Forum 527-529, p. 559-562 (2006). 